Research Article

\title{
Utilizing Technology Acceptance Model for Influences of Smartphone Addiction on Behavioural Intention
}

\author{
Chih-Wei Lin $\mathbb{D}$, ${ }^{1}$ Yu-Sheng Lin $\mathbb{D}^{2},{ }^{2}$ Chia-Chi Liao $(\mathbb{D}){ }^{3}$ and Chih-Cheng Chen $\mathbb{D}^{4,5}$ \\ ${ }^{1}$ Department of Leisure Services Management, Chaoyang University of Technology, Taichung 413310, Taiwan \\ ${ }^{2}$ Physical Education Office, Chaoyang University of Technology, Taichung 413310, Taiwan \\ ${ }^{3}$ Department and Graduate Institute of Applied English, Chaoyang University of Technology, Taichung 413310, Taiwan \\ ${ }^{4}$ Department of Automatic Control Engineering, Feng Chia University, Taichung 40724, Taiwan \\ ${ }^{5}$ Department of Aeronautical Engineering, Chaoyang University of Technology, Taichung 413, Taiwan
}

Correspondence should be addressed to Chia-Chi Liao; liao.chiachi@icloud.com and Chih-Cheng Chen; ccc@gm.cyut.edu.tw

Received 31 January 2021; Accepted 16 May 2021; Published 1 June 2021

Academic Editor: Cheng-Fu Yang

Copyright ( $\odot 2021$ Chih-Wei Lin et al. This is an open access article distributed under the Creative Commons Attribution License, which permits unrestricted use, distribution, and reproduction in any medium, provided the original work is properly cited.

\begin{abstract}
The purpose of this study was to establish a behavioural model of students' smartphone usage based on the perception of new advanced information technology, perceived playfulness, and smartphone addiction (SA). Undergraduate students were chosen to be the participants from a university in Taiwan. There were 814 valid questionnaires and the valid response rate was $81.4 \%$. Firstly, SA positively and significantly affected perceived playfulness, perceived ease of use, and perceived usefulness. Secondly, perceived usefulness did not significantly influence behavioural intention. However, there was an unforeseen result; the effect of SA on perceived usefulness was barely significant. It can be concluded that the participants in the present study were undergraduate students and they might not intend to use smartphones for their academic performance. The findings indicated that undergraduate students experienced perceived playfulness, perceived ease of use, and perceived usefulness of smartphone from their addiction perception, which further implied that smartphone usage was interesting, easy, and useful. It was inferred that the undergraduate students were already under a high technology addiction (TA) condition. Suggestions indicate that the conjunction of teaching and mobile application should be extensively applied. It should be based on students' dependence on smartphone and smartphone's enjoyment to helpfully improve teaching via smartphones.
\end{abstract}

\section{Introduction}

The technology acceptance model (TAM) [1] is designed to explore the effects of external variables on perceived usefulness (PU), perceived ease of use (PEOU), attitude towards usage (A), behavioural intentions (BI), and actual behaviour $(\mathrm{AB})$; the purpose is to discuss user's acceptance or rejection of using new information technology with two internal beliefs, which are PU and PEOU, and predict user's A accurately. In TAM, PU is defined as using a specific system which would improve a person's performance at work; PEOU is described as using a particular system which would be effortless [2].

Fishbein and Ajen [3] pointed out that attitude is a result that comes from past learning experiences. Users consistently have either likable or dislikeable behaviour on a specific thing; namely, attitude is an overall individual evaluation of specific people or a thing. In light of behavioural intention, Davis, Bagozzi, and Warshaw [1] defined BI as the users' willingness to continuously use a specific system or recommend it to others. An individual's subjective consciousness would decide the possibility of using information technology in the future as his/her AB. Davis [2] and Ahn et al. [4] have shown that PEOU positively affects PU. The connection between PEOU and PU on attitude towards usage shows that the outcomes are worth learning and affective cognition influences user's efficacy to perform [1].

According to $\mathrm{Lu}$, Zhou, and Wang [5], the instant messaging software is a fast and convenient tool for communication and it is easy to use. It has attracted many users, 
especially young people. Notably, users have a higher perception of usefulness and ease of use with regard to new technology or new service and his/her attitude towards using would tend to be positive. A is a complement to BI that originates from a positive effect [1]. Lin, Yang, Sia and Tang [6] proposed a smartwatch study, and the results indicated that it positively and significantly affected BI. Users believe that a smartwatch is worth using and his/her willingness is decided by his/her affective consciousness. According to mobile payment research (Lin et al., [7]; August), the researchers illustrated that PU and PEOU positively influenced BI. Users will have a high willingness to use a mobile system if they experience virtual mobile service on a smartphone with ease. Besides, the results of previous studies referring to the Internet, Facebook, instant messaging, and smartphone usage have shown a positive relationship between PU and BI [5, 8-10].

We now turn to the evidence on perceived playfulness. In 2001, Moon and Kim illustrated that the typical TAM does not extremely predict user's motivation; they indicated that much easier IT usage from computer systems will be regarded as a pleasant human-computer interaction [11]. Hence, they proposed a connection between PEOU and perceived playfulness (PP) when using the World Wide Web (www). Based on this, they divided PP into three dimensions: concentration (CON), curiosity (CUR), and enjoyment (ENT). They claimed that the easier the information technology is, the higher intention from the state of playfulness will be considered. According to the research results of user-created content services [12], playfulness is the main factor which determines a user's BI.

Chen, Gillenson, and Sherrell [13] indicated that PP plays a significant role in cyberspace and mobile services; it can stimulate a user's BI. Furthermore, Lin et al. [7] explained that PP, PEOU, and PU of smartwatch usage all positively influence A. Given that PP significantly affects A and BI when developing a new technology system, it further influences subsequent usage behaviour [14-17]. With regard to smartphone addiction (SA), the understanding of SA to date is similar to Internet addiction [18]. Griffiths [11] illustrated that Internet overuse can be regarded as pathological Internet usage or technological addiction. With the diverse features of smartphone, more and more young people have become dependent on or addicted to the smartphone functions. They not only are addicted to sending SMS via smartphones but also rely on other tools of the smartphone [19]. Khang, Kim, and Kim [20] defined SA as digital media addiction; the longer time a user spends on it, the higher the addiction to cyberspace. Leung and Wei [21] found that mobility, immediacy, and functionality were the main motivations that can be used to predict mobile user behaviour. When a user is not satisfied with using his/her smartphone, it can result in the asymmetry of usage time verse usage motive. Simultaneously, an individual's SA may distort his/her internal beliefs, which further increases smartphone usage time.

According to the results of an online auction addiction (eBay users) [22] and social networking websites addiction (Facebook users) [23], the level of addiction strengthens users' PP perception. In other words, user's recognition of addiction is distorted by the level of how he/she is addicted to technology. Moreover, according to the results of eBay research, the level of online auction addiction significantly deepens the perception of usefulness and playfulness but slightly influences PEOU [22]. Additionally, concentration [15] shows that a user concentrates on a specific activity with playfulness and he/she ignores external interferences and cannot realise how fast time flies. That being said, SA can be described as a user's overly reliance on a smartphone and he/ she further exhibits the unable to withdrawal symptom. Addiction is a mental condition that originated from substance abuse and substance dependence which results in overuse. Technology addiction (TA) is a new mental addictive situation which has been incorporated with different technology media. Turel, Serenko, and Giles [22] indicated that TA is a special type of behavioural addiction, which is a psychological dependency on IT usage, which twists the user's perceptions of usefulness, enjoyment, and ease of use towards the system and which makes users become addictive. According to Serenko and Turel [23], a user from a different technology web portal shows different TAs. Additionally, the result showed that Facebook users demonstrate higher TA symptoms than eBay users [22].

1.1. The Purpose of the Study. The TAM [1] has been widely used as the theoretical basis in various research fields. However, there are not enough studies using SA as the antecedent variable to strengthen PP, PEOU, and PU. Moreover, there is no direct relationship with attitude from the abovementioned TA example. Given that, the main purpose of the present study is to bridge the gap and use the expanded TAM to test TA with regard to $\mathrm{PP}, \mathrm{PEOU}$, and $\mathrm{PU}$ on the smartphone which immerse users in cyberspace. Besides, participants of previous studies mainly focused on high school students. Balakrishnan and Raj [24] indicated that undergraduate students are a veritable group of high-risk Internet addiction. Accordingly, the present study developed a SA model and validated the relationships among all the research variables to understand the effects of university students' perceptions of smartphone usage (PP, PEOU, and PU) on $\mathrm{BI}$ through a mediator of positive attitude as shown in Figure 1.

1.2. The Importance of the Study. Nowadays, people have become more dependent on their smartphone. This phenomenon may lead to SA. This study has adopted the TAM to test PP, PEOU, and PU of a smartphone which engages undergraduate students with the surroundings. Most importantly, SA in the present study was an antecedent variable that deepens PP, PEOU, and PU. Moreover, the research data of PP, PEOU, and PU which made the participants be addicted to smartphone the most were analysed and the relationships among all variables were tested so as to comprehend the influence.

1.3. Limitations. First of all, owing to human resource limitation, the respondents were selected from a university in central Taiwan by purposive sampling. Hence, the 


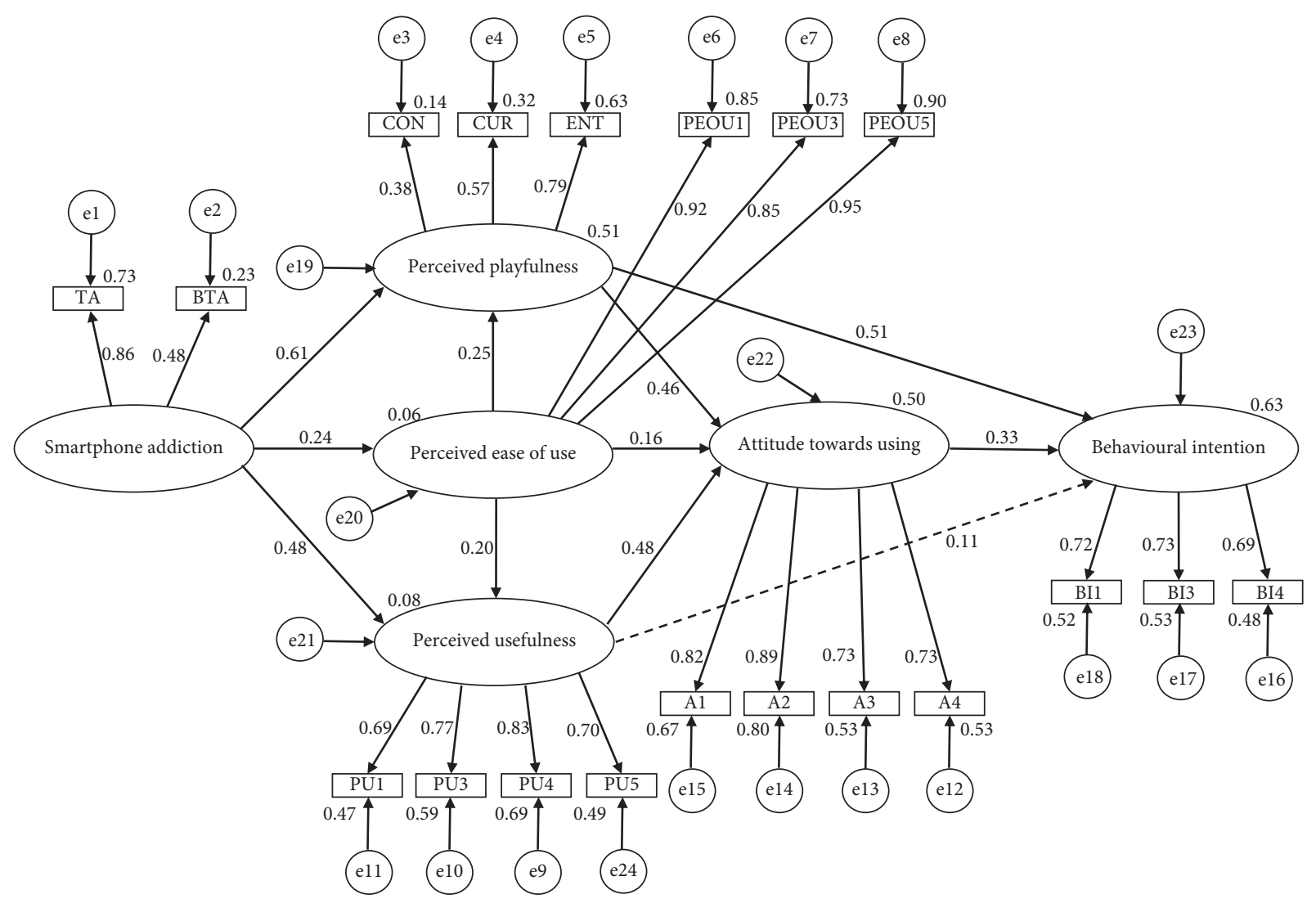

FIGURE 1: Path map of a smartphone addiction (SA) behavioural model. There is no significant difference between perceived usefulness (PU) and behavioural intention (PI) (dashed line).

research data in the present study cannot represent all Taiwanese students. Secondly, the definition of PP, PEOU, and PU does not specifically focus on the same usage motivation. Therefore, the research variable in the present study cannot represent all Taiwanese students. Finally, the function of a smartphone is changed with the advancement in science and technology; hence, the research data in the present study cannot be used to represent all smartphone functions in the future.

\section{Method}

The study was conducted via an online questionnaire survey which was adopted by purposive sampling. The undergraduate students who had smartphones with individual mobile Internet programmes were chosen. One thousand questionnaires were delivered randomly from a university in Taiwan. In total, 186 questionnaires were excluded due to incompleteness or incorrect answers, resulting in a sample of 814 adolescents (81.4\% of valid responses). Participants were university students $(N=814$; 201 male and 613 female); the students from 22 different departments were asked to participate. The introduction of the study was explained at first and the research motivation clarified that the questionnaire was designed to measure students' recognition of playfulness, ease of use, and usefulness that were related to their smartphone. There were 38 questions in total in this questionnaire and it took
5-10 minutes to complete. All participants were told that all comments will only be used for academic research, not open to the public.

2.1. Measures. Each scale consisted of five items, which were responded to on a 5-point Likert scale ranging from 1 (strongly disagree) to 5 (strongly agree). Reliability and validity were tested by confirmatory factor analysis (CFA). Firstly, for SA, the TA scale [23] was adapted to measure students' perceptions of TA and behavioural technology addiction (BTA). The factor loading for SA was from 0.61 to 0.78 ; composite reliability (CR) was 0.86 ; and average variance extracted (AVE) was 0.46. Secondly, for PP, the www context scale [15] was adapted for students' perceptions of CON, CUR, and ENT. The factor loading for PP was from 0.41 to 0.93 ; CR was 0.91 ; and AVE was 0.54 . Thirdly, for PEOU and PU, the innovative use of smartphone scale [9] and mobile services quality scale [25] were used to assess students' perceptions of smartphone's ease of use and usefulness at school for academic performance. The factor loading for PEOU was from 0.85 to 0.98 ; CR was 0.93 ; and AVE was 0.83 . The factor loading for PU was from 0.67 to 0.84; CR was 0.83; and AVE was 0.56. Finally, for A and BI, the www context scale [15] and innovative use of smartphone scale [9] were used to measure students' perceptions of positive and negative evaluations and future willingness. The factor loading for A was from 0.71 to 0.92 ; CR was 0.87 ; 
and AVE was 0.63 . The factor loading for BI was from 0.69 to 0.77; CR was 0.77; and AVE was 0.52.

2.2. Data Analysis. Firstly, descriptive statistics was used to understand the distribution of demographic information. This study used the frequency allocation table, percentage, average, and standard deviation to explore the current situation of TAM, PP, and SA. Furthermore, CFA and structural equation model (SEM) with SPSS AMOS 23 were used. Model adequacy checking was evaluated by using absolute fit indices, relative fit indices and parsimonious fit indices which contain root mean square residual (RMR), goodness of fit index (GFI), error of approximation (RMSEA), comparative fit index (CFI), normed fit index (NFI), parsimonious normed fit index (PNFI), chi-square $\left(\chi^{2}\right)$ /degree of freedom $(\mathrm{d} f)$ ratio, and Hoelter's critical $\mathrm{N}$ (CN). A cut-off value greater than 0.90 was for GFI, CFI, and NFI; a cut-off value lesser than 0.50 was for the RMR; a cutoff value greater than 0.50 was for the PNFI; cut-off values lesser than 0.08 or greater than 0.05 were for the RMSEA, indicating acceptable model fit [26]; a cut-off value lesser than 5 was for the chi-square $\left(\chi^{2}\right) /$ degree of freedom $(\mathrm{d} f)$ ratio; and a cut-off value greater than 200 was for the $\mathrm{CN}$.

\section{Results}

3.1. Sample Distribution. There were 814 participants in total, out of which 201 were male $(24.7 \%)$ and 613 were female participants (75.3\%). In light of the division, over 700 respondents $(86 \%)$ studied in day school. The education background of most of the participants was freshman (195, $24 \%)$. As for academy, 327 (40.2\%) respondents studied in College of Management. In terms of department, there were 22 departments in this university. The highest response was from the "Department and Graduate Institute of Finance," with 60 people $(7.4 \%)$. Conversely, the lowest one was the "Department of Landscape and Urban Design," with 7 people $(0.9 \%)$.

SEM was used to examine a SA behavioural model with direct paths. Firstly, the paths were from the dimensions of SA (TA and BTA) to PP, PEOU, and PU. Secondly, the paths were from PP (CON, CUR, and ENT), PEOU, and PU to A. Thirdly, the paths were from PEOU to PP and PU. Finally, the paths were from PP (CON, CUR, and ENT), PU, and A to BI. Besides, the indirect effects from the dimensions of SA on BI were also examined. After each variable model was examined, the results showed that SA indirectly influenced $\mathrm{BI}$ via the mediators of PP, PEOU, and PU. The structural model exhibited a good model of fit. Given that, the final SA behavioural model and standardised coefficients are shown in Figure 1; the fit test indices are shown in Table 1.

Table 1 shows that PEOU and BI were saturated; PNFI values of PU and A were lower than 0.5 . Besides, $\chi^{2} / \mathrm{d} f$ value of each variable was lower than 5; GFI value, CFI value, and NFI value of each variable were higher than 0.9 ; RMR value of each variable was lower than 0.05; RMSEA value of each variable was lower than 0.08 ; $\mathrm{CN}$ value of each variable was higher than 200, which reached the standard. Overall, the
TABLE 1: Fit test indices.

\begin{tabular}{lcccccccc}
\hline Variable & RMR & GFI & RMSEA & CFI & NFI & PNFI & $\chi^{2} / \mathrm{d} f$ & CN \\
\hline PU & 0.01 & 0.99 & 0.07 & 0.99 & 0.99 & 0.33 & 5.30 & 460 \\
A & 0.01 & 0.99 & 0.06 & 0.99 & 0.99 & 0.33 & 4.26 & 572 \\
PP & 0.03 & 0.97 & 0.06 & 0.97 & 0.96 & 0.64 & 4.04 & 306 \\
SA & 0.04 & 0.98 & 0.06 & 0.98 & 0.97 & 0.60 & 3.85 & 363 \\
Overall & 0.05 & 0.98 & 0.02 & 0.99 & 0.98 & 0.74 & 1.26 & 646 \\
\hline
\end{tabular}

Note. PEOU and BI were saturated.

goodness of fit test of the SA behavioural model conformed to the standard.

Figure 1 and Table 2 display that A directly affected BI (with a path coefficient of 0.330 ). Moreover, PP directly and indirectly influenced BI. However, PU only indirectly affected BI (with a path coefficient of 0.116). Oum and Han [12] indicated a nonstatistically significant relationship between PU and BI; the result supports the notion that there is no significant difference between PU and BI (with a path coefficient of 0.110 ). Thirdly, PEOU indirectly affected BI via A, PP, and PU. Finally, SA indirectly influenced BI through $\mathrm{PP}, \mathrm{PEOU}$, and PU. Besides, comparing three independent variables of the total effect on $\mathrm{BI}$, the most powerful independent variable was PP with an effect of 0.662 , followed by PEOU with 0.263; the weakest one was PU with 0.226. However, as for the effect of the antecedent variable (SA) on $\mathrm{BI}, \mathrm{PP}$ was the most influential with the indirect effect of 0.404, whereas PU was the weakest with 0.038 .

\section{Discussion}

Firstly, according to Table 3, PEOU was the highest item, indicating that the undergraduate students could mostly use their smartphone with no difficulty. Hence, ease of use of smartphone could be an influential factor which helps a student improve his/her life or task. Moreover, different perceptions of ease of use were found. For example, PEOU1 represented that most of the participants could use their smartphone effortlessly. On the contrary, PEOU3 represented that most of the undergraduate students could use their smartphone skilfully.

Secondly, the total effects on PU towards the undergraduate students were satisfactory. Notably, the undergraduate students generally felt that their smartphone was useful. Therefore, the usefulness of smartphone could be an influential factor which helped the student enhance his/her life or task. In addition, the highest item PU5 illustrated that smartphones made the participants feel helpful, whereas the lowest item PU1 indicated that improving their academic performance via smartphone was acceptable. Thirdly, the total cognitions on A pointed out that undergraduate students had a positive and pleasant attitude when using their smartphones. Besides, A3 with the highest score showed that smartphone usage made the respondents feel pleasant. On the contrary, A4 was the lowest one, showing that there was a moderate recognition of positive idea for using smartphones. The undergraduate students mostly assented to participate in using smartphones again. Furthermore, BI3 showed that the participants had a mid-to-high cognition of 
TABLE 2: Direct effects, indirect effects, and total effects of each variable on behavioural intention.

\begin{tabular}{|c|c|c|c|c|c|c|}
\hline \multirow[t]{9}{*}{ Antecedent variable } & \multicolumn{2}{|c|}{$\begin{array}{l}\text { Independent } \\
\text { variable }\end{array}$} & \multirow{2}{*}{$\begin{array}{c}\text { Mediator variable } \\
\mathrm{A}\end{array}$} & \multirow{2}{*}{$\begin{array}{c}\text { Direct effect } \\
0.330\end{array}$} & \multirow[t]{3}{*}{ Indirect effect } & \multirow{2}{*}{$\begin{array}{c}\text { Total effect } \\
0.330\end{array}$} \\
\hline & & \multirow{4}{*}{-} & & & & \\
\hline & \multirow{3}{*}{$\mathrm{PP}$} & & & 0.510 & & 0.662 \\
\hline & & & $\mathrm{A}$ & & 0.152 & \\
\hline & & & A & & 0.053 & 0.263 \\
\hline & \multirow[t]{2}{*}{ PEOU } & PP & & & 0.165 & \\
\hline & & PU & & & 0.045 & \\
\hline & \multirow{2}{*}{ PU } & - & & 0.110 & & 0.226 \\
\hline & & & A & & 0.116 & \\
\hline \multirow{3}{*}{ SA } & & PP & & & 0.404 & 0.505 \\
\hline & & PEOU & & & 0.063 & \\
\hline & & $\mathrm{PU}$ & & & 0.038 & \\
\hline
\end{tabular}

TABLE 3: Average and standard deviation of each variable $(n=814)$.

\begin{tabular}{lccc}
\hline Variable & Item/aspect & Mean & SD \\
\hline \multirow{3}{*}{ PEOU } & PEOU1 & 4.43 & 0.66 \\
& PEOU3 & 4.35 & 0.72 \\
& PEOU5 & 4.42 & 0.67 \\
& Overall & 4.40 & 0.64 \\
PU & PU1 & 3.26 & 0.84 \\
& PU3 & 3.60 & 0.97 \\
& PU4 & 3.29 & 0.93 \\
& PU5 & 3.74 & 0.82 \\
& Overall & 3.47 & 0.73 \\
A & A1 & 3.84 & 0.76 \\
& A2 & 3.69 & 0.80 \\
& A3 & 3.88 & 0.80 \\
& A4 & 3.50 & 0.81 \\
BI & Overall & 3.73 & 0.67 \\
& BI1 & 3.78 & 0.84 \\
& BI3 & 3.79 & 0.85 \\
& BI4 & 3.41 & 0.94 \\
PP & Overall & 3.73 & 0.67 \\
\hline \multirow{4}{*}{ SA } & CON & 3.08 & 0.78 \\
& CUR & 3.44 & 0.73 \\
& ENT & 3.86 & 0.70 \\
& Overall & 3.46 & 0.55 \\
\hline & TA & 3.62 & 0.70 \\
& BTA & 2.81 & 0.87 \\
& Overall & 3.16 & 0.68 \\
\hline
\end{tabular}

frequently using smartphones in the future. BI4 was with the lowest but a medium score.

Fourthly, the overall effects on PP were moderate. Besides, the highest ENT showed that the playfulness of smartphones could make the undergraduate students feel happy, whereas the lowest CON indicated that the undergraduate students were conscious when using smartphones. Finally, the overall average of SA was moderate and the undergraduate students had a mid-to-low-end recognition of TA. In other words, the undergraduate students had awareness of smartphone usage and they could restrict themselves from being addicted. In addition, TA was higher than BTA, which represented that the meaning of TA could make better impressions on the participants. However, the overall average of TA and BTA was not ideal. It was inferred that the items of TA originated from a compulsive buying tendency. People may have salience, withdrawal, conflict, relapse, and mood modification symptoms when shopping online. Moreover, SA in the present study was used to test the undergraduate students who may have the same symptoms when using smartphones. Therefore, the undergraduate students may have a higher recognition for TA to answer TA. Furthermore, it was assumed that the items of BTA originated from the eBay environment and were used to test for shopping addiction. People may have obsessivecompulsive disorder while online shopping and might be sensitive about the price. Nevertheless, SA in the present study was not used to test the undergraduate students' purchasing power via their smartphones. Therefore, the undergraduate students may have a lower recognition for BTA and the items may not be completely suitable for answering TA. Thus, it was suggested that TA and BTA should be tested separately in the future.

4.1. Mediator Effects of PP, PEOU, and PU on BI via $A$. Attitude was influenced by PP, PEOU, and PU in the present study; it is a mediator variable which affects BI. Besides, the results indicated that $\mathrm{PP}, \mathrm{PEOU}$, and $\mathrm{PU}$ indirectly influenced BI through A. It can be inferred that undergraduate students are pleasant and satisfied when they are using their smartphones; they are relatively pleased and have active playfulness of their smartphone. A joyful smartphone recreation function may enhance the pleasure in students' life and strengthen the degree of devoting time to their life. Therefore, the most influential direct variable was PP with an effect of 0.510 . However, the effect of PP on BI via A was 0.152 as shown in Table 2. It was assumed that the diversified developments of mobile applications combine with smartphone enjoyment so as to enhance the user's dependence on smartphones. Additionally, PEOU and PU directly influenced $A$ with an effect of 0.16 and 0.35 , respectively. These results are supported by the notion that was proposed by Lin et al. [7].

Nevertheless, both PEOU and PU adjusted BI through A; the outcomes showed a slightly indirect effect (PEOU with an effect of 0.263 and PU with 0.226 ). It was concluded that university students spend one-third of their time in the learning environment at school. However, the case of 
teaching via smartphone function by teachers is rare. Therefore, university students cannot properly recognise the practical utility of smartphones in their life or learning environment. Moreover, education in Taiwan has recently popularised online learning, distance learning, massive open online courses, flipped classroom, and digital teaching materials compiled on campus in order to integrate with smart learning. It encourages people to learn unlimitedly through the technical function of smartphones or tablets.

4.2. Effects of $S A$ on $P P, P E O U$, and $P U$. The results verified that a higher SA perception enhances a smartphone's performance, especially playfulness; it leads the undergraduate students to appreciate their smartphone and further continue to use their smartphone. Besides, it was inferred that the undergraduate students were already under a high TA condition before they sensed PP, PEOU, and PU. However, the level of SA did not totally deepen PP, PEOU, and PU. The effects of SA on PU were slightly significant. It was concluded that TA of mobile device users and TA of cyberspace users may exhibit different TA symptoms from different using motivations. Notably, the level or the influence of TA should be determined by the IT feature. With regard to $\mathrm{PP}$, it is always the main factor of using cyberspace or technology product $[13,15]$. When a user has a cognitive bias by overusing or abusing smartphone, his/her dependent behaviour will result in addictive symptom. In light of this, PP was shown to be the most powerful variable in this study; the result supported the notion that PP has the strongest effect than PEOU and PU. If smartphone usage cognition has a positive relationship with BI, the students will believe that the smartphone is beneficial and they will use their smartphones carefully. As pointed out in the introduction of this paper, there is a significant connection among PP, PEOU, and PU. The higher PP, PEOU, and PU are, the higher $\mathrm{BI}$ is. As expected, the results support this standpoint. The outcome in this study explained that total effect of SA on PP, PEOU, and PU was 0.505 as shown in Table 2.

According to online auction addiction [22] and social networking websites addiction [23], both studies explained that addiction distorts users' perceptions of usefulness and enjoyment. However, the effect of addiction on PEOU was weak [22]. Serenko and Turel [23] indicated that social networking website addiction distorted technology perceptions. Comparing the two results, it can be seen that using social networking website is easier than using online auction website. Basically, Facebook provides its users with a hedonic platform, whereas online auction website provides more complex information (e.g., payment process). Hence, it was concluded that social networking website users have a stronger potential to show TA symptoms. Based on the previous findings, the notions proposed by Turel et al. [22] and Serenko and Turel [23] are supported. Due to the different IT usages, the model of using smartphone properly, to find the solutions or use smartphone improperly to solve the problem, was verified in this study. Finally, in the present study, the results support the notion that addiction positively influences PP, PEOU, and PU.

\section{Conclusions and Theoretical Implications}

The result of this study showed that SA successfully mixed with the TAM model. The purpose of this study was to comprehend what SA stood for when it was combining with the TAM model. Moreover, the results verified that higher SA perception enhanced smartphone's performance, especially playfulness; it led the undergraduate students to have an appreciation on their smartphone and further continue to use their smartphone. However, SA should be efficiently faced; otherwise, it would distort the way people interact with technology systems. The undergraduate students perceived PP, PEOU, and PU of smartphone from their addiction perception which further caused them to feel smartphone using that was interesting, easy, and useful. According to research results, it was inferred that the undergraduate students were already under a high TA condition before they sensed PP, PEOU, and PU perception. Keep using smartphone that results in physical problems.

In the present study, the level of SA did not totally deepen the perceptions of PP, PEOU, and PU. The effects of SA on PU were slightly significant. Comparing with Facebook addiction [23], the addiction results were different. Hence, it was concluded that TA of $3 \mathrm{C}$ mobile device user and TA of cyberspace user may exhibit different TA 114 symptoms from different using motivations. Namely, the level or the influence of TA should be determined by the IT feature. Moreover, the influences of PEOU and PU on smartphone in the present study were different from the TAM model. The results showed that PU could not directly affect BI; it had to be decided via attitude towards using. Namely, there was a disagreement between undergraduate students' subjective appraisal of performance on smartphone and smartphone itself. However, the TAM model combining with SA enhanced the effects from antecedent variable. Hence, for multimedia materials teaching class, special attention should be paid to students who may have a symptom of addiction.

\section{Suggestions}

(1) This study focused on the effects of SA on behavioural intention. It is suggested that future studies should first investigate smartphone usage's purpose from the participants; it may be helpful for future researches to enlarge the research range so as to obtain more accurate research data.

(2) It was recommended that future studies should collect the questionnaires from different universities or different cities in Taiwan in order to propose different viewpoints of SA on behavioural intention.

(3) It is suggested that SA caused by playfulness from the university students should be weakened, whereas the perception of dependence on using smartphone in their life and learning environment should be strengthened. 
(4) It is proposed to upgrade the Internet facilities on campus and allow teachers to practice digital teaching materials and other related study courses.

\section{Data Availability}

All data generated or analyzed during this study are included within this manuscript and are available from the corresponding author upon request.

\section{Conflicts of Interest}

The authors declare that they have no conflicts of interest.

\section{Acknowledgments}

The work was partially supported by the National Science Council of Taiwan under Grant MOST 107-2221-E-507-002MY3.

\section{References}

[1] F. D. Davis, R. P. Bagozzi, and P. R. Warshaw, "User acceptance of computer technology: a comparison of two theoretical models," Management Science, vol. 35, no. 8, pp. 982-1003, 1989.

[2] F. D. Davis, "Perceived usefulness, perceived ease of use, and user acceptance of information technology," MIS Quarterly, vol. 13, no. 3, pp. 319-340, 1989.

[3] M. Fishbein and I. Ajzen, Belief, Attitude, Intention, and Behavior: An Introduction to Theory and Research, AddisonWesley, Reading, MA, USA, 1975, https://www.researchgate. net/publication/233897090_Belief_attitude_intention_and_ behaviour_An_introduction_to_theory_and_research.

[4] T. Ahn, S. Ryu, and I. Han, "The impact of web quality and playfulness on user acceptance of online retailing," Information \& Management, vol. 44, no. 3, pp. 263-275, 2007.

[5] Y. Lu, T. Zhou, and B. Wang, "Exploring Chinese users' acceptance of instant messaging using the theory of planned behavior, the technology acceptance model, and the flow theory," Computers in Human Behavior, vol. 25, no. 1, pp. 29-39, 2009.

[6] C. W. Lin, C. C. Yang, W. Y. Sia, and K. Y. Tang, "Examining the success factors of smart watch: a behavioral perspective on consumers," Polish Journal of Management Studies, vol. 20, 2019.

[7] C. W. Lin, S. S. Lee, K. Y. Tang, Y. X. Kang, C. C. Lin, and Y. S. Lin, "Exploring the users behavior intention on mobile payment by using TAM and IRT," in Proceedings of the 2019 3rd International Conference on E-Society, E-Education and E-Technology, pp. 11-15, Taipei Taiwan, August 2019.

[8] S. Lee and M. Cho, "Social media use in a mobile broadband environment: examination of determinants of twitter and facebook use," International Journal of Mobile Marketing, vol. 6, no. 2, pp. 71-87, 2011.

[9] Y. Park and J. V. Chen, "Acceptance and adoption of the innovative use of smartphone," Industrial Management \& Data Systems, vol. 107, no. 9, pp. 1349-1365, 2007.

[10] T. S. H. Teo, V. K. G. Lim, and R. Y. C. Lai, "Intrinsic and extrinsic motivation in internet usage," Omega, vol. 27, no. 1, pp. 25-37, 1999.
[11] M. Griffiths, "Does internet and computer "addiction" exist? some case study evidence," CyberPsychology \& Behavior, vol. 3, no. 2, pp. 211-218, 2000.

[12] S. Oum and D. Han, "An empirical study of the determinants of the intention to participate in user-created contents (UCC) services," Expert Systems with Applications, vol. 38, no. 12, pp. 15110-15121, 2011.

[13] L.-D. Chen, M. L. Gillenson, and D. L. Sherrell, "Enticing online consumers: an extended technology acceptance perspective," Information \& Management, vol. 39, no. 8, pp. 705-719, 2002.

[14] R. Agarwal and E. Karahanna, “Time flies when you're having fun: cognitive absorption and beliefs about information technology usage," MIS Quarterly, vol. 24, no. 4, pp. 665-694, 2000.

[15] J.-W. Moon and Y.-G. Kim, "Extending the TAM for a worldwide-web context," Information \& Management, vol. 38, no. 4, pp. 217-230, 2001.

[16] H. Van Der Heijden, "User acceptance of hedonic information systems," MIS Quarterly, vol. 28, no. 4, pp. 695-704, 2004.

[17] J. Zhang and E. Mao, "Understanding the acceptance of mobile SMS advertising among young Chinese consumers," Psychology and Marketing, vol. 25, no. 8, pp. 787-805, 2008.

[18] M. Kwon, J. Lee, W. Won et al., "Development and validation of a smartphone addiction scale (SAS)," PLoS One, vol. 8, no. 2, pp. 1-7, 2013.

[19] M. C. Bian, "Linking psychological attributes to smart phone addiction," Hong Kong, China, The Chinese University of Hong Kong, 2012, http://pg.com.cuhk.edu.hk/pgp_nm/ projects/2012/BIAN\%20Mengwei\%20Casey.pdf.

[20] H. Khang, J. K. Kim, and Y. Kim, "Self-traits and motivations as antecedents of digital media flow and addiction: the internet, mobile phones, and video games," Computers in Human Behavior, vol. 29, no. 6, pp. 2416-2424, 2013.

[21] L. Leung and R. Wei, "More than just talk on the move: uses and gratifications of the cellular phone," Journalism \& Mass Communication Quarterly, vol. 77, no. 2, pp. 308320, 2000.

[22] O. Turel, A. Serenko, and P. Giles, "Integrating technology addiction and use: an empirical investigation of online auction users," MIS Quarterly, vol. 35, no. 4, pp. 1043-1062, 2011.

[23] A. Serenko, O. Turel, and O. Turel, "Integrating technology addiction and use: an empirical investigation of facebook users," AIS Transactions on Replication Research, vol. 1, pp. 1-18, 2015.

[24] V. Balakrishnan and R. G. Raj, "Exploring the relationship between urbanized Malaysian youth and their mobile phones: a quantitative approach," Telematics and Informatics, vol. 29, no. 3, pp. 263-272, 2012.

[25] F. B. Tan and J. P. C. Chou, "The relationship between mobile service quality, perceived technology compatibility, and users' perceived playfulness in the context of mobile information and entertainment services," International Journal of Human-Computer Interaction, vol. 24, no. 7, pp. 649-671, 2008.

[26] L. T. Hu and P. M. Bentler, "Cutoff criteria for fit indexes in covariance structure analysis: conventional criteria versus new alternatives," Structural Equation Modeling: A Multidisciplinary Journal, vol. 6, no. 1, pp. 1-55, 1999. 